\title{
Simulation of the evacuation process at various economic facilities using the Anylogic software product
}

\author{
Marina Avdeeva ${ }^{1 *}$, Oleg Uzun ${ }^{1}$, and Yulia Borodkina ${ }^{1}$ \\ ${ }^{1}$ Peter the Great St. Petersburg Polytechnic University, Polytechnicheskaya str., 29, 195251, St. \\ Petersburg, Russia
}

\begin{abstract}
The article deals with the problem of evacuation of people in case of emergency situations. Modeling of the process of evacuation of people from premises is quite new and one of the most promising tools for planning the organization of pedestrian flows in emergency situations, for predicting problems arising during the evacuation of people, and ultimately for saving human lives. The experimental model was developed using the AnyLogic simulation tool. An office space was chosen as the object of research, where the evacuation mode was simulated and the experiment was conducted. The average evacuation time of each person and the total exit time, as well as the intensity of the human flow at the entrance and exit of the building are calculated. A hypothesis about the influence of time of day on the duration of evacuation was put forward and proved. It is concluded that the built model provides the necessary information for predicting the fire situation in office premises and, as a result, the possibility of their elimination, as well as in agricultural planning and Economics.
\end{abstract}

\section{Introduction}

In a big city, a person has to be in crowded places every day: public transport, at crowded events, at catering points, at the workplace. This fact creates the problem of mass evacuation of people in case of emergency $[8,9]$.

Undoubtedly, conducting real experiments in this task requires significant financial and organizational difficulties. From which we can identify the importance of modeling the above processes to determine the preferred room geometry and the emergence of stable groups of human clusters, which will allow us to most effectively, from the standpoint of the number of lives saved, form an emergency response process for emergencies and their further elimination $[3,4]$.

Simulation of the process of evacuating people from premises is a proven and one of the most effective means for planning the organization of pedestrian flows in emergency situations, to anticipate problems encountered during the evacuation of people, and ultimately to save lives [10].

\footnotetext{
*Corresponding author: avdeeva_mo@spbstu.ru
} 
The basic principles and principles of the theory of the movement of human flows are quite well described by V.V. Kholshchevnikov, V.M. Predtechensky, A.I. Milinsky, A.P. Parfenenko [12], in whose works the problems of movement in buildings and in the urban environment are studied, addresses the key issue of emergency evacuation of people [3] and their free movement [7].

A significant contribution to the development of simulation modeling was made by the scientist V.D. Boev. He was engaged in the development of the Anylogic software product, on the basis of which he wrote more than 180 scientific papers. However, in his research V.D. Boev did not use the pedestrian library, as his interest was directed to the study of other areas of simulation [2].

\section{Formulation of the problem}

The relevance of the article is due to the need to improve the evacuation process in a safe and reliable way. The authors of this article set the task of simulating the evacuation of employees from the office space in the form of a black box, which is shown in Figure 1.

The source data also uses the plan for the evacuation of the real office and the daily routine. Based on the task, a solution to this problem can be implemented in the class of simulation models $[11,13]$.

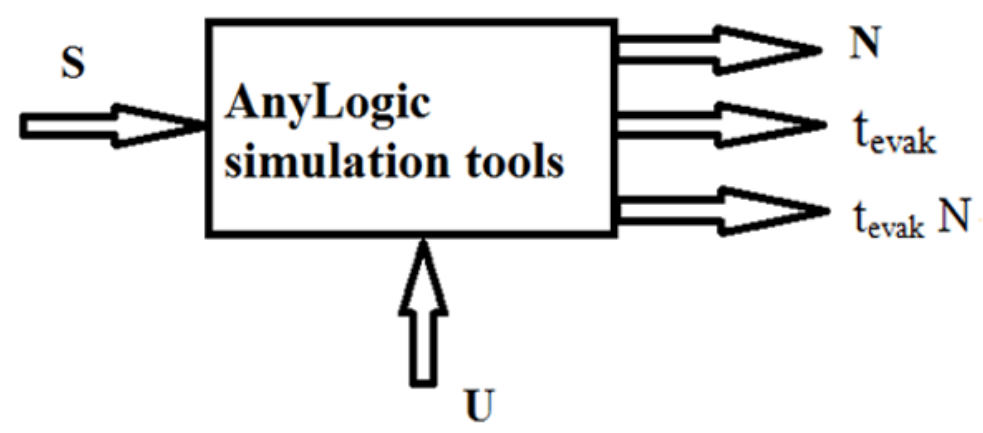

Fig. 1. Statement of the problem of modeling the evacuation of office workers, where, $S$ - the number of employees in the office space, $\mathrm{N}$ - the number of evacuated people, tevak - total time of evacuation of all employees, tevak $\mathrm{N}$ - average time of evacuation of the $\mathrm{N}$-th employee, $\mathrm{U}$ - the control variable, for which we will use the time of day.

Simulation allows you to bring the model as close as possible to the real situation and conduct a series of experiments, which is extremely important for predicting the course of the evacuation process, modeling possible scenarios and preventing human casualties $[5,6]$. The collective behavior of people when moving in dense human flows has become an important direction in the study of the evacuation process [1], when agent models occupy a special place in the simulation process [9].

\section{Methods}

As an effective platform, we will use the Anylogic software package, which has a developed pedestrian library and a wide range of tools for collecting statistical results of the experiment and allows us to fully implement the agent approach.

In the designed model, it is necessary to determine the behavior of each agent and its interaction with the environment and other agents. After studying the existing methods for modeling pedestrian flows, the agent method was chosen, which allows not to describe the 
whole system, but to set the rules that each agent must obey. To create an office building model in the Anylogic simulation tool, rules can be set using special libraries.

The authors of this article decided to use the Pedestrian Library, which is used to model pedestrian flows. Rules for agents are set using the blocks of the pedestrian library (Figure 2).

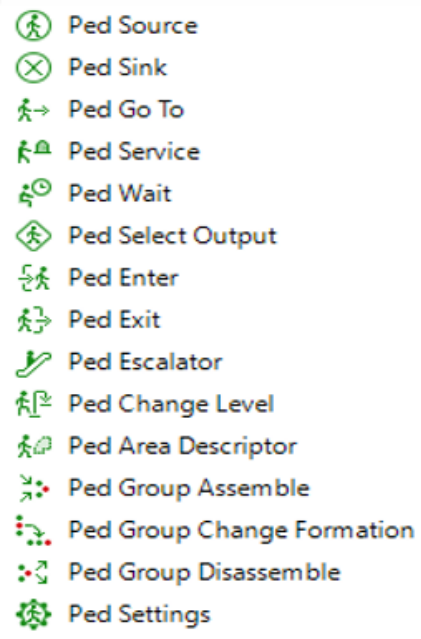

Fig. 2. Blocks of the pedestrian library.

The blocks selected and connected in a certain sequence create an algorithm (scheme) of people's behavior when various events occur. Figure 3 shows the logic of the model of movement of people in a building in normal mode.

The general view of the model before starting is shown in Figure 4. In addition, a clock, a people counter in the building, an alarm button and graphs are added to the animation window to display statistics on the results of evacuation simulations.

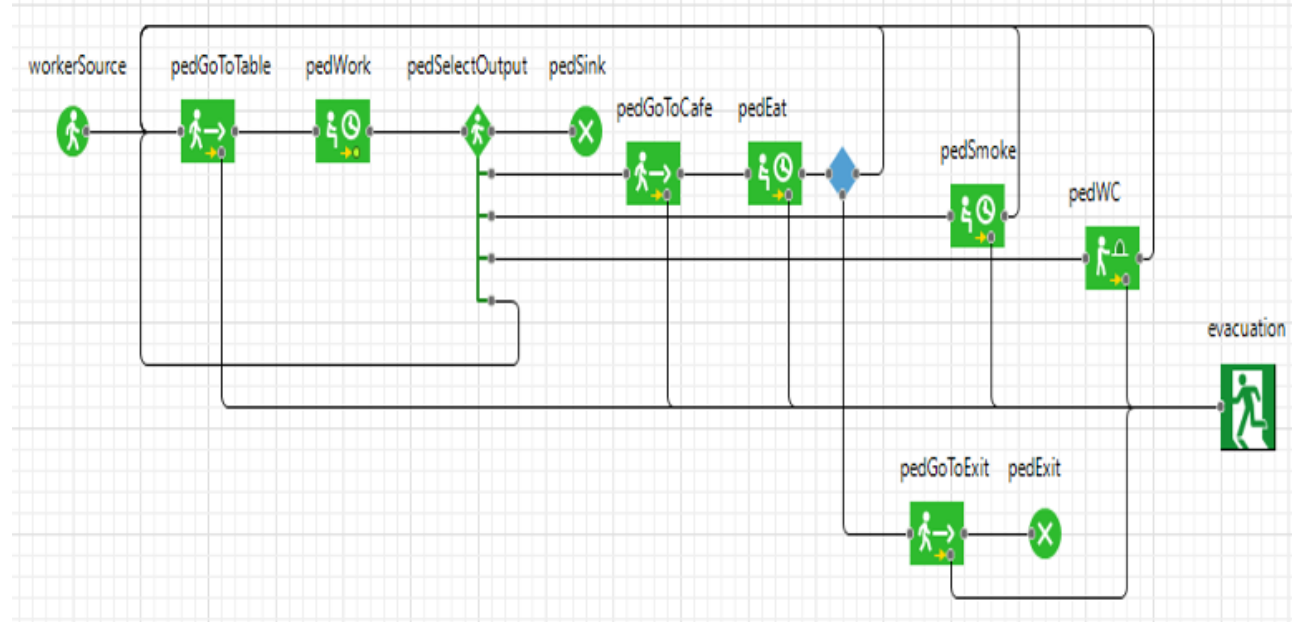

Fig. 3. The movement of workers. 


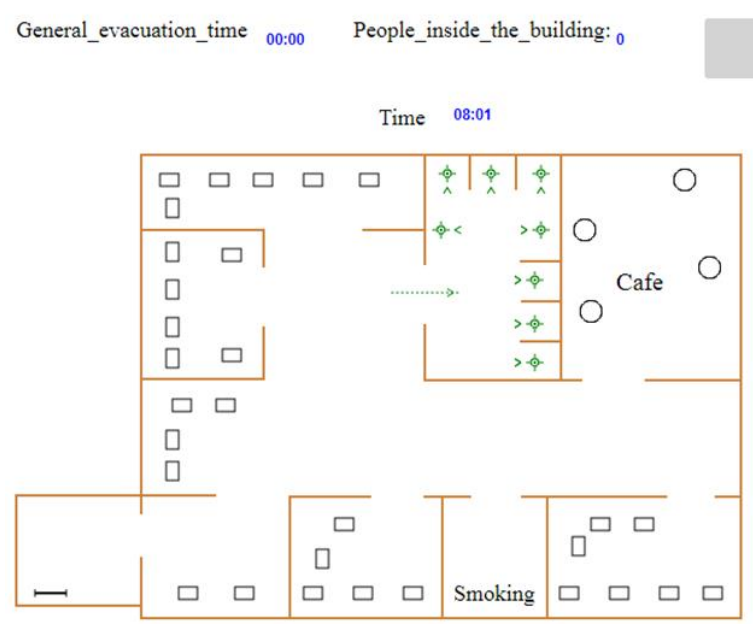

\section{FIRE!!!}

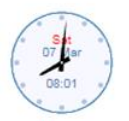

Statistics of modeling results

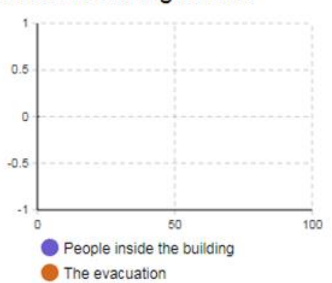

The evacuation

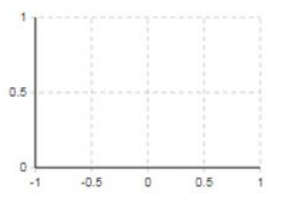

The average duration of the evacuation

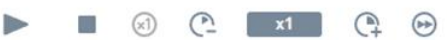

A Пауза [马]

Fig. 4. General view of the model before starting.

\section{Experiment Results}

On the constructed model, the authors of the article conducted a series of experiments, the results of which are shown in the figures below.

Figure 5 displays a visualization of a workflow at a random point in time.

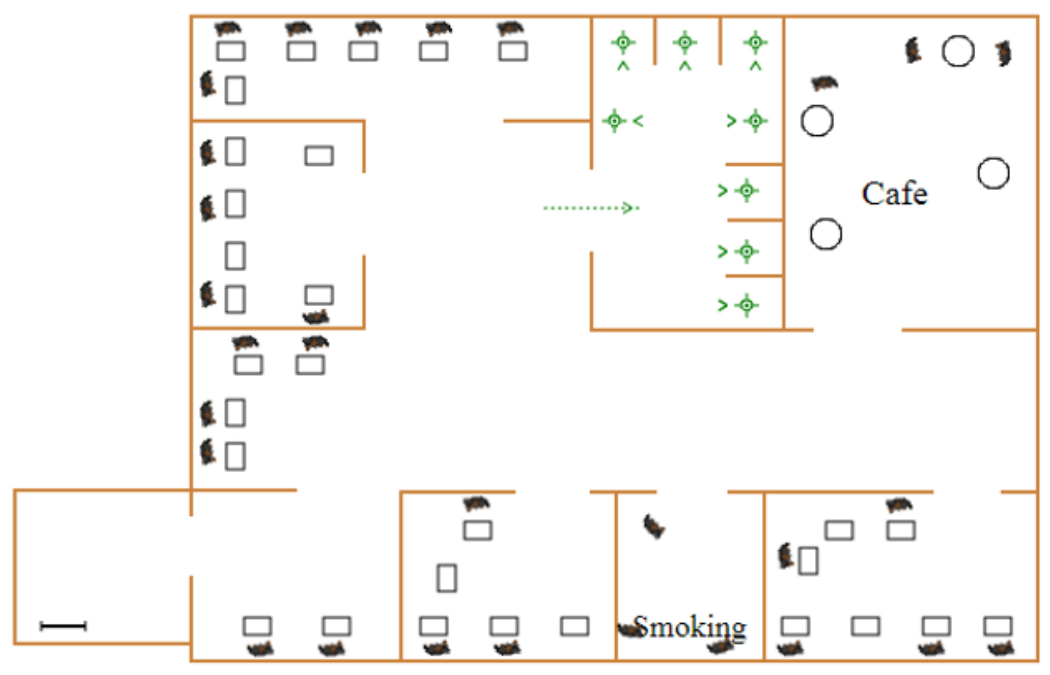

Fig. 5. Visualization of a workflow at a random point in time.

At the beginning of the evacuation, 27 people are in the room. Figure 6 shows the process of leaving the building by workers and the numerical indicators of the process 2 minutes after clicking on the alarm. 


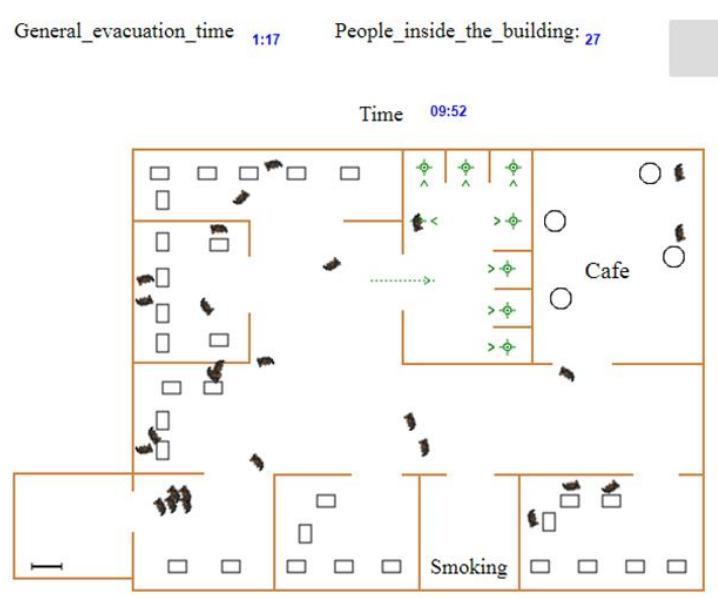

\section{FIRE!!!}

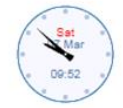

Statistics of modeling results

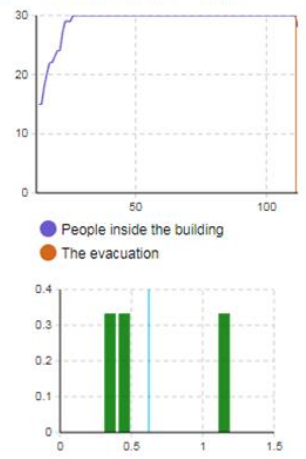

The average duration of the evacuation 0.62

II

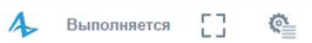

Fig. 6. Start of the evacuation process.

The animation window in Figure 7 shows the final stage of the evacuation simulation. After 4 minutes 44 seconds after the start of the evacuation, the last person left the building, the timer stopped.

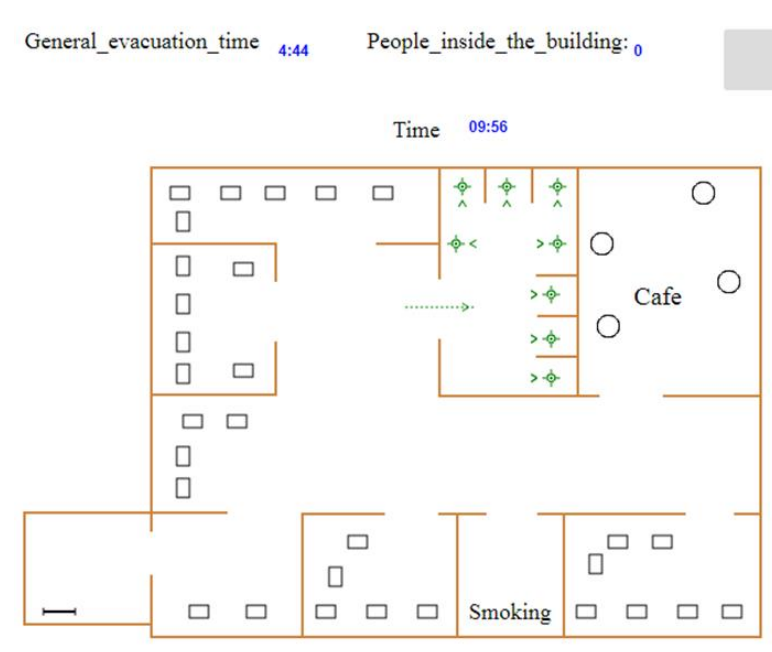

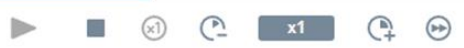

\section{FIRE!!!}

Statistics of modeling results

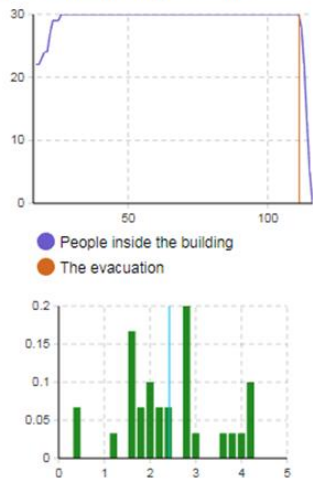

The average duration of the evacuation 2.42

Fig. 7. The end of the evacuation process.

The evacuation process reflects statistics of simulation results. The graph of the number of people after the linear section abruptly begins to fall, and the number of people in the building again reaches zero. The second graph describes the average duration of the evacuation, which is numerically equal to 2 minutes and 42 seconds. 


\section{Conclusions}

Next, we test the hypothesis that evacuation during the day takes on average longer than in the morning. To test the dependence of the evacuation duration on the time of day, 10 simulations were performed in the morning and afternoon. Based on this experiment, conclusions can be drawn about the properties of the object described by the mathematical model.

The alarm button gave a signal at a random time, and the evacuation duration for each case was calculated using the model parameters. Table 1 presents the experimental results. As we can see, the average evacuation time in the afternoon is 31 seconds longer than in the morning.

Table 1. Duration of evacuation depending on the time of day.

\begin{tabular}{|c|c|c|c|c|c|c|c|c|c|c|c|}
\hline \multirow{2}{*}{ № } & \multicolumn{10}{c|}{ Durtion of evacuation, sec } \\
\cline { 2 - 13 } & 1 & 2 & 3 & 4 & 5 & 6 & 7 & 8 & 9 & 10 & t av. \\
\hline morning & 4.17 & 4.13 & 4.51 & 4.32 & 4.26 & 4.21 & 4.30 & 4.37 & 4.20 & 4.53 & 4.30 \\
\hline day & 4.46 & 4.07 & 4.24 & 5.28 & 4.14 & 4.19 & 5.11 & 4.52 & 5.05 & 5.03 & 4.61 \\
\hline
\end{tabular}

A computer experiment confirmed that in the described model, the duration of evacuation from the building depends on the time of day. There are several reasons for this.

In the first hour of work, employees are mainly at workplaces, in the middle of the day they can walk in different rooms. When the evacuation begins, people are lost, spend time returning for things, dressing, etc. Thus, the dependence of the evacuation duration on the time of day is experimentally confirmed in the described model.

The model built by the authors also allows you to record the intensity of the human flow at the entrance and exit of the office space. To measure the intensity, the "flow Statistics" element was added to the graphic editor. This element allows you to collect statistics on the intensity of pedestrian flows that pass through a given line in the simulated space. Figure 8 shows a complete picture of the intensity of people moving through the entrance. The first section describes the beginning of the working day and the arrival of all employees in the office-this explains the jump in the schedule. Then the intensity drops to zero and continues its "zero movement" until the evacuation begins. After the fire alarm is triggered, all employees tend to leave the premises, resulting in a sharp increase in the intensity of the flow of people.

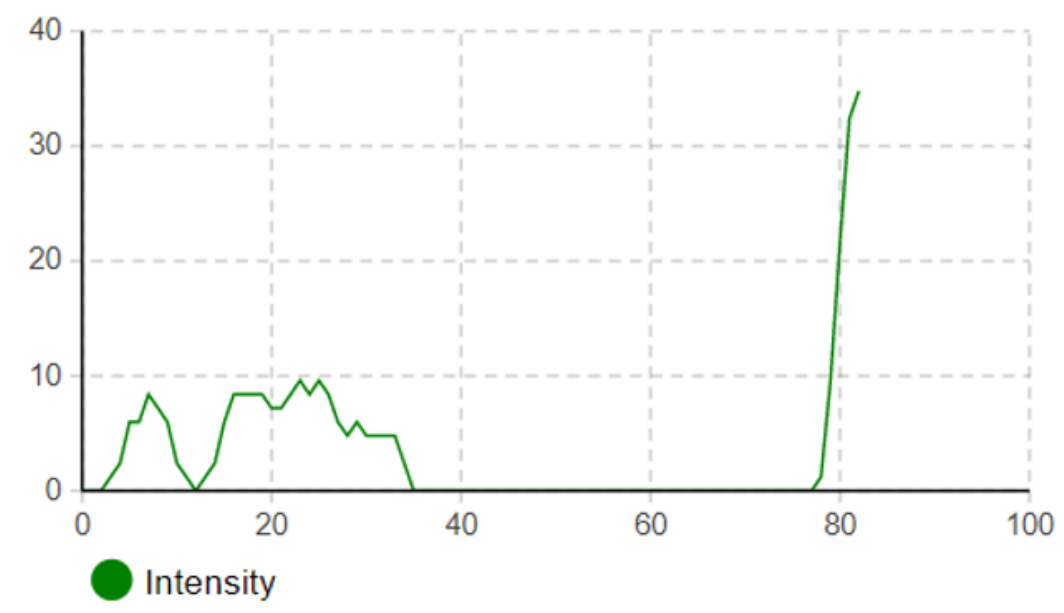

Fig. 8. Intensity of the human flow at the entrance/exit to the building. 
Conclusions are:

1. Simulation of the fire situation on the built model of an office space was Carried out.

2. Simulation results are Obtained that allow us to quantify the output parameters of the model described in the problem statement: the number of people evacuated, the time of evacuation, the intensity of the flow of people.

3. Based on the experiments conducted on the built model of office space, it can be concluded that the use of simulation techniques greatly facilitates the task of conducting a large number of experiments, allows you to obtain adequate results, as well as predict various situations that can reduce human losses and in some situations prevent their death.

\section{References}

1. A.M. Aptukov, D.A. Bratsun, Mechanics, 3, 18-23 (2009)

2. V.D. Boev, Simulation Systems (Moscow, 2017) https://www.biblioonline.ru/bcode/407617

3. G. Chu, J. Wang, Q. Wang, Structural Safety, 38, 22-31 (2009) https://doi.org/10.1016/j.strusafe.2012.02.001

4. G.Q. ChuT, T. Chen, Z.H. Sun, J.H. Sun, Building and Environment, 42(3), 12831290 (2007) https://doi.org/10.1016/j.buildenv.2005.12.002

5. J. Xin, Ch. Huang, Fire Safety Journal, 62, 72-78 (2013) https://doi.org/10.1016/j.firesaf.2013.09.022

6. V.V. Kholshchevnikov, Human flows in buildings, structures and in the territories of their complexes (Moscow, 1983)

7. V.V. Kholshchevnikov, Vestnik TGASU, 2, 89-103 (2011)

8. V.V. Kholshchevnikov, D.A. Samoshin, Evacuation and behavior of people during fires (Academy of State Fire Service of the Ministry of Emergencies of Russia, Moscow, 2015)

9. C. Macal, M. North, Journal of Simulation, 4, 151-162 (2010)

10.R.F. Malikov, Workshop on the simulation of complex systems in AnyLogic 6 (Ufa, 2013)

11.M. Tancogne-Dejean, P. Laclémence, Fire Safety Journal, 80, 9-19 (2016) https://doi.org/10.1016/j.firesaf.2015.11.009

12.A.P. Parfenenko, Fire and explosion safety, 23(12), 46-53 (2014)

13.G. Ramachandran, Fire Safety Journal, 2, (1979/80) 125-145. https://doi.org/10.1016/0379-7112(79)90039-0. 